\title{
El diseño de tareas de entrenamiento en el fútbol desde el enfoque de la pedagogía no lineal Design of training tasks in football from the nonlinear-pedagogy approach
}

Alberto Martín-Barrero, Pablo Camacho Lazarraga

Centro Universitario San Isidoro (España)

\begin{abstract}
Resumen: El diseño de tareas y su planificación ha emergido como uno de los principales enfoques en las líneas de investigación sobre aquellos investigadores que apoyan la bases conceptuales de la pedagogía no lineal como elemento clave para el proceso de enseñanzaaprendizaje y el entrenamiento. Es por ello, que cada vez más se investiga cómo afectan los diferentes constreñimientos en relación a los comportamientos y patrones de acción de los jugadores, tanto en el plano individual como colectivo. Se realiza una propuesta práctica con diferentes niveles de complejidad, en las que se manipulan la aleatorización de los movimientos de los jugadores, el grado de oposición, las fases del juego, el rol del jugador y la forma de gol. Esta nueva visión de la pedagogía no lineal, nos obliga a replantearnos el concepto de tarea de entrenamiento, orientándolo hacia un entorno dinámico adaptado a las necesidades de los jugadores. El objetivo de este trabajo es realizar una propuesta práctica a través de diferentes contextos de aprendizaje (tipos de tareas) de menor complejidad, manipulando/ constriñendo ciertos elementos de la misma, con el fin de aumentar el grado de variabilidad y complejidad, a través de estrategias que mejoren el proceso de adaptación del jugador a las situaciones reales de juego.
\end{abstract}

Palabras clave: diseño de tareas, entrenamiento, pedagogía no lineal, constreñimiento, fútbol.

\begin{abstract}
The design and planning of tasks has emerged as one of the main focuses in the lines of research for those researchers who support the conceptual basis of nonlinear pedagogy as a key element for the teaching-learning process and training. For this reason, more and more research is being carried out on how the different constraints affect behaviors and action patterns of the different players, both individually and collectively. A practical proposal is made with different levels of complexity, in which the randomization of players' movements, the degree of opposition, the phases of the game, players' role, and goal forms are manipulated. This new vision of nonlinear pedagogy pushes us to rethinking the concept of training tasks, directing it towards a dynamic environment adapted to players' needs. The objective of this work is to carry out a practical proposal through different learning contexts (types of tasks) of less complexity, manipulating / constraining certain elements of it, in order to increase the degree of variability and complexity, through strategies that improve players' adaptation process to real game situations.
\end{abstract}

Key words: task design, training, nonlinear pedagogy, constraints, soccer.

\section{Introducción}

La ciencia del deporte permite a los técnicos deportivos reflexionar, adquiriendo nuevos conocimientos y herramientas que mejoren el proceso de entrenamiento de los deportistas. En los últimos años, la investigación y el estudio de la didáctica y la metodología del deporte ha permitido que emerjan nuevas propuestas y tendencias en el entrenamiento deportivo, tales como la pedagogía no lineal. Uno de los aspectos más importante que transmite este nuevo enfoque es la necesidad de entender la acción deportiva como una relación de adaptación jugador-entorno y no como un proceso mecánico de ejecución precedido por un modelo a seguir. Entender este nuevo enfoque es importante, ya que la psicología ecológica nos indica que el comportamiento humano no puede analizarse ajeno al contexto en el cual este tiene lugar (Gibson, 1979). Además el proceso de aprendizaje y de mejora en el rendimiento del jugador no viene determinado por la suma divisible de las diferentes cualidades del deportista, sino por un proceso de auto-organización que permite optimizar las diferentes capacidades y habilidades del deportista (Flores-Rodríguez, 2019). Este nuevo enfoque enfatiza la importancia que tienen los deportistas en entornos que proporcionan oportunidades de acción o «affordances» (Del Villar \& García, 2014; Passos, Araujo \& Shuttleworth, 2008; Warren, 2006).

Este nuevo enfoque difiere sustancialmente de las

Fecha recepción: 23-11-19. Fecha de aceptación: 31-03-20

Alberto Martín-Barrero

amarbar10@gmail.com metodologías de enseñanza más tradicionales, basadas en la repetición de patrones cerrados de movimiento y donde la toma de decisiones se limitan a contextos y situaciones cerradas. La pedagogía no lineal se está consolidando como una alternativa importante, tratando de proporcionar un marco teórico que proporcione una profunda comprensión sobre los deportes de invasión y de equipo (Clark, Mcewan, \& Christie, 2018; Balagué, Torrents, Pol, \& Seirul·lo, 2014). Esta nueva corriente de la pedagogía no lineal se basa en la comprensión del alumno, el entorno del aula, el profesor y el proceso en sí como un sistema dinámico no lineal y complejo (Torrents, Araujo, Gordillo \& Vives, 2011), donde los jugadores perciben los estímulos directamente del entorno real y los entrenadores manipulan las variables moderadoras que puedan influir en la resolución de la tarea, ampliando con ello las fuentes de información que guían a los jugadores hacia la consecución de sus objetivos, con objeto de que sean los jugadores los que, sin ningún intermediario y basado en sus propias acciones, extraiga de forma autónoma la información relevante en cada situación concreta y actúe en consecuencia.

Este trabajo tiene como objetivo mostrar desde una perspectiva práctica las propuestas de la pedagogía no lineal, en un deporte de máxima incertidumbre como el fútbol. Par ello se partirá desde el análisis de la dinámica del juego y su lógica interna, que será el punto de partida y la base que justificará y determinará las principales características que se deben tener en cuenta en la configuración de las tareas de entrenamiento en el fútbol. 


\section{Características del fútbol desde la lógica interna y su relación con la configuración de las tareas}

Tal y como menciona Parlebás (2001) Entendemos por lógica interna a las características principales o rasgos pertinentes de una determinada situación motriz, que configura las condiciones, prescripciones y relaciones en las que cada participante puede jugar o actuar (Lagardera \& Lavega, 2003).

A finales de los años 80 se superaba el concepto de comportamiento motor, para pasar a la comprensión de la conducta motriz como reflejo de la persona que actúa con una intencionalidad. Ello llevó a entender las conductas motrices unidas a la implicación total de la persona que actúa (Larraz Urgelés, 1989). Es por ello por lo que surgen estudios que, a través de la teoría de la acción motora de Parlebas (1976; 1981; 1988), estudian el comportamiento motor de la persona teniendo en cuenta no solo las variables motoras, sino además, la dimensión cognitiva y emocional. El campo de la praxiología motriz de Parlebas $(1976 ; 1981 ; 1988)$ por tanto, representa una teoría que se fundamenta en una perspectiva sistémica que trata conceptos tan importantes en nuestro deporte como lo son la 'comunicación motriz', la 'interacción motriz', el 'rol sociomotor' y la 'lógica interna' en el juego. Según esta teoría, podemos afirmar que las posibilidades de acción de los jugadores vendrán determinadas por el tipo de rol a el comportamiento motor de la persona y sub-rol que adopten, su valencia, nivel de ajuste, así como de otras variables presentes durante una acción de juego (Pic, Lavega-Burgués \& March-Llanes, 2019), es decir, dependiendo de la interacción que se produzca entre todos estos elementos, los jugadores adoptarán un comportamiento motor específico (Parlebás, 1981; 1996). La regularidad en estos comportamientos también dependen de la estructura de comunicación a la que los jugadores están sujetos, siendo estos comportamientos una forma ordenada de comunicarse en los juegos con reglas (Pic \& Navarro, 2017; Caldeira, Paulo, Infante \& Araujo, 2019).

Siguiendo la definición expuesta en las líneas anteriores y relacionándola con el fútbol, podemos determinar que éste, es un deporte de cooperación o colectivo, donde una serie de jugadores interaccionan para conseguir un objetivo común. Pero este objetivo común está condicionado por la presencia de adversarios u oponentes, los cuales además tienen un carácter invasor, es decir, pueden invadir el espacio de juego del rival. A su naturaleza motriz podemos añadir que es un juego en el cual se puede usar cualquier parte del cuerpo, siendo los pies el principal elemento anatómico que permite desarrollar la actividad deportiva. Además, dentro de su estructura formal, podemos distinguir diferentes elementos que le dan un carácter específico: metas (porterías), móvil (balón), espacio de juego (terreno de juego), reglas de juego (determinadas por la normativa de la FIFA). También, es importante considerar que tiene una funcionalidad que parte desde los principios del juego hasta los diferentes medios técnico-tácticos.

La interacción de estos elementos proporcionan al fútbol unos principios que determinan su dinámica como juego. Estos principios o reglas intentan configurar y definir su complejidad y están compuesto por cuatro principios (Martín-Barrero, 2019):
- Principio de especificidad: define las características propias del juego en sí, sus elementos formales y características motrices.

- Principio de inestabilidad: el fútbol es un juego que está en constante estado de inestabilidad por el continuo cambio en sus fases y momentos del juego, lo que determina un cambio constante en los roles de los jugadores.

- Principio de incertidumbre: debido a la presencia e invasión de adversarios se produce una variación constante de las condiciones y las situaciones espacio/temporales, conteniendo un importante grado de variabilidad.

- Principio de interacción: el juego está definido por la comunicación constante entre los jugadores de un mismo equipo según el poseedor del móvil. Lo que conlleva una constante relación entre los diferentes subsistemas, jugadores y rivales. Además hay que añadir que la interacción de jugadores y las capacidades de los mismos conlleva la aparición de cualidades emergentes. También es importante la interacción con la competición y su influencia en los comportamientos de equipo (expulsiones, amonestaciones etc.).

Podemos considerar que a través del conocimiento y la comprensión de estos principios podremos optimizar el proceso de entrenamiento, intentando trasladar cada uno de ellos, a las diferentes tareas que realizamos durante las sesiones de entrenamiento. La pregunta es, ¿cómo podemos transferir estos aspectos conceptuales al entrenamiento y por el ende al proceso de aprendizaje y mejorar del rendimiento deportivo? Las diferentes propuestas que parten de la pedagogía no lineal, nos indican que los comportamientos emergen de la interacción del jugador con la tarea de entrenamiento y que estos comportamientos o conductas pueden ser fomentadas o provocadas a través de los constreñimientos o «constrains» (Chow., Davids, Button, Shuttleworth, Renshaw, \& Araújo, 2007).

\section{Propuesta práctica}

En la siguiente propuesta práctica se seleccionarán los dos tipos de tareas que menos aproximación tienen en relación a los principios de la dinámica del juego (inespecífica y genérica), aumentando de forma gradual la incertidumbre, inestabilidad, especificidad e interacción de los elementos que lo componen. El proceso consistirá en seleccionar una tarea de las características mencionadas anteriormente, manipular los constreñimientos de la tarea y hacer una comparativa entre la versión original (V.O) y la versión final(V.F.). La leyenda de los iconos que se utilizarán para la representación gráfica es la siguiente (Figura 1).

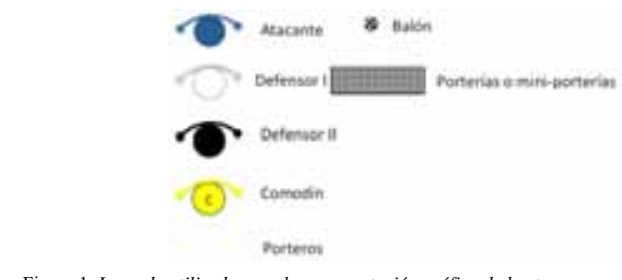

Figura 1. Leyenda utilizada para la representación gráfica de las tareas.

En la primera propuesta práctica (Tabla 2) se propone un tipo de tarea de carácter inespecífica, donde el grado de complejidad es muy bajo. Esta tarea consiste en encadenar una secuencia de pases desde el primer hasta el último jugador. 
Dicha secuencia es marcada por el entrenador antes del inicio de la tarea. Para aumentar la complejidad de la tarea se utilizarán los siguientes constreñimientos (tipo de tarea) y a la vez se describirá la conducta provocada:

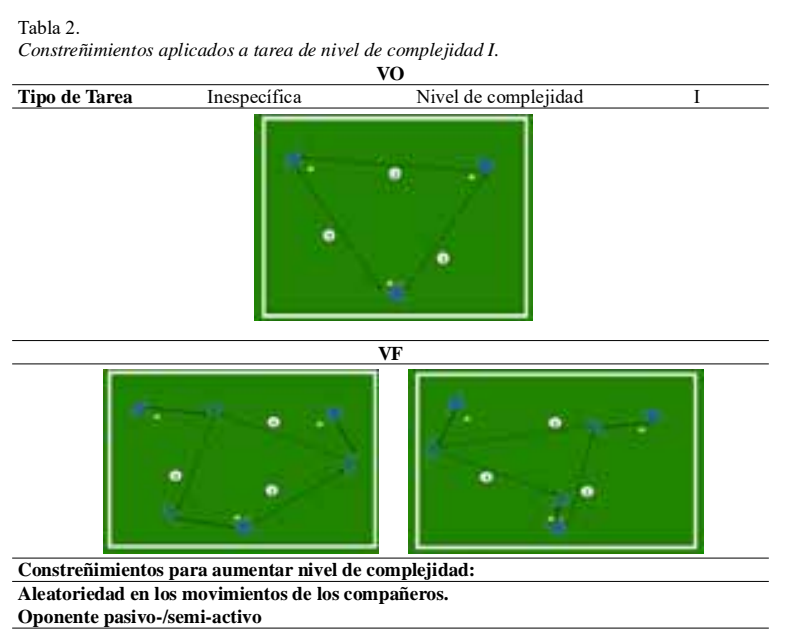

- Aleatoriedad en los movimientos o cambios en las posiciones de inicios en los practicantes (Passos et al. 2008): de los jugadores que hacen de receptores del balón, teniéndose que desmarcar en diferentes planos y ángulos y nunca repitiendo el anterior movimiento. Comportamiento provocado: condiciona el tipo, la distancia, la velocidad y la dirección del pase del pasador con respecto al receptor.

- Oponente pasivo/semi-activo: presencia de jugadores que realizan otro tipo de acciones y que perturban el espacio de acción del pasador. Si queremos aumentar el nivel de dificultad y por ende el nivel de complejidad, se puede proponer una defensa semi-activa, caracterizada por una presencia más cercana e intensa pero sin llegar a robar. Comportamiento provocado: con respecto al pasador, mismos aspectos que el anterior constreñimiento, con respecto al receptor, ofrecer un desmarque o línea de pase dependiendo de la presencia del oponente pasivo y orientación del control con respecto al mismo.

Estos dos constreñimientos incrementan el nivel de incertidumbre de la tarea, ya que dichos elementos aumenta la variabilidad de respuestas de los jugadores y la toma de decisiones en relación a un elemento concreto del juego (presencia de rivales y acción en relación a su comportamiento, además de la respuesta en relación a la acción de un compañero). Como se observan en la representación gráfica (Tabla 2) de la evolución de la tarea, en la VF podemos ver como la variabilidad en el desmarque de los jugadores receptores provoca también variabilidad en los diferentes pases, condicionando constantemente la distancia, velocidad y superficie de golpeo.

Para la segunda propuesta práctica (Tabla 3) el tipo de tarea elegido es de la de carácter genérica, en la cual el nivel de complejidad aumenta en relación a la propuesta en el ejemplo anterior. Esta tarea consiste en una conservación de balón entre dos equipos, un atacante y un defensor más dos comodines. Para aumentar la complejidad de la tarea se propondrá los siguientes constreñimientos:

Transición de fases y cambios de roles: introducción de mini-porterías para dar continuidad al juego y provocar que el equipo que realiza la conservación de balón pase a defen- der las mini-porterías y el equipo que defiende intente meter gol en las mismas una vez recuperada la posesión del balón. Provocar cambios de roles y fases entre atacantes y defensores. Comportamiento provocado: jugadores del equipo que atacan cuando pierden el balón tienen que pasar a defender y viceversa, completando siempre el ciclo de juego (ataquedefensa) y adaptándose a la inestabilidad del mismo.

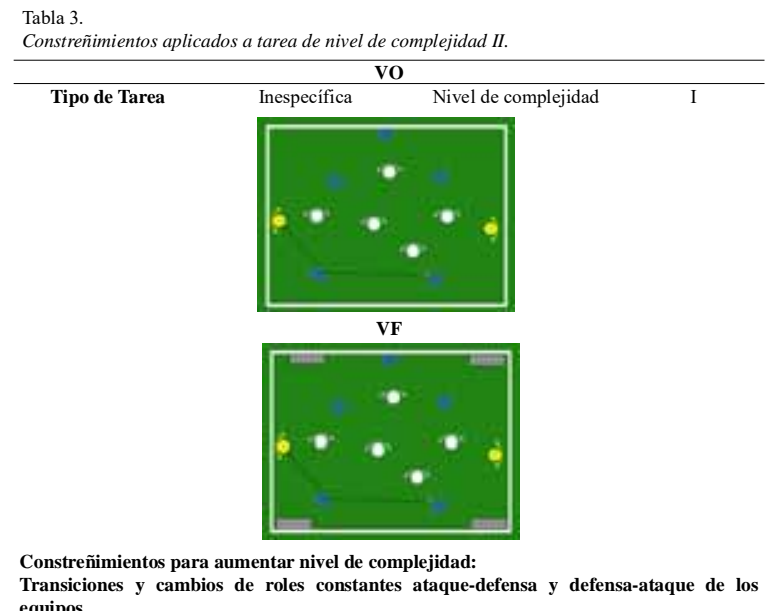

Transiciones y cambios de roles constantes ataque-defensa y defensa-ataque de los equipos.

En la tercera propuesta práctica (Tabla 4) se relacionará con el principio de interacción, entre los comportamientos deseados y como condicionarlos a través de la interacción del jugador con el sistema de puntuación. Se utilizará una tarea de carácter modificada a través de una situación reducida de 6x6 jugadores con un sistema 1-3-1-1.

Condicionar la forma del gol: se conseguirá gol doble si el mismo es conseguido por una de las zonas siguientes: raso cerca de uno de los palos o por la escuadra. Comportamiento provocado: finalizar a gol buscando las zonas de la portería donde resulta más difícil alcanzar el balón para el portero.

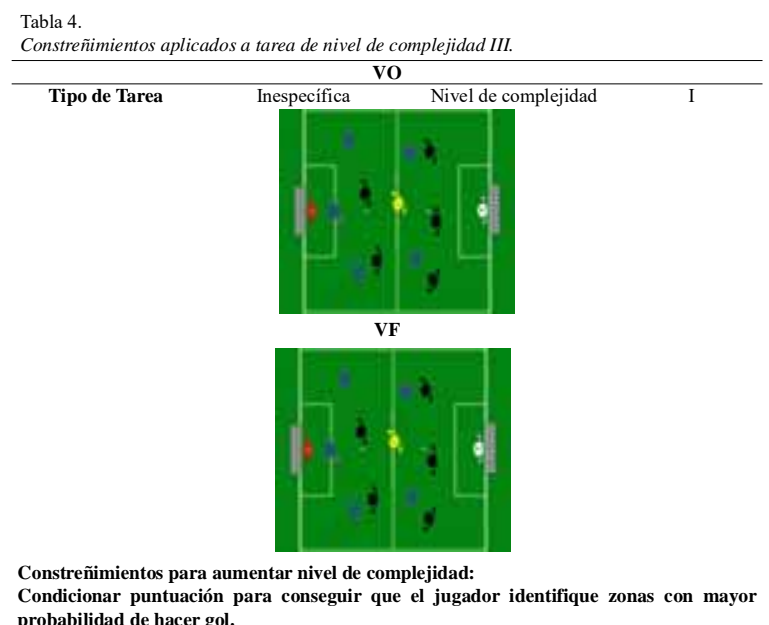
probabilidad de hacer gol.

\section{Resultados}

Los principales resultados que se obtienen de la experiencia realizada nos indican que, en líneas generales, la manipulación de los constreñimientos del entorno determina las posibilidades de acción de los jugadores, promoviendo determinados comportamientos en base a las interacciones de todas las variables que configuran las acciones de juego. 


\section{Disausión}

Son varios los estudios que recientemente han analizado, en los deportes de equipo, la configuración de las tareas y las diferentes variables pedagógicas que intervienen en las mismas (Gamero-Portillo, García-Ceberino, Reina, FeuMolina \& Antúnez-Medina, 2019; Reina, García-Rubio, Antúnez \& Ibáñez, 2019) que influyen La limitación de comportamientos o constreñimientos y su gestión en el diseño de tareas juegan un papel fundamental en la aparición de nuevas conductas y por lo tanto en el proceso de aprendizaje. Para ello, es importante tener en cuenta que el deportista debe tener un rol activo, que permita desarrollar patrones adaptativos al contexto (en este caso la tarea de entrenamiento) y alejándose de los modelos tradicionales que tratan de imponer un modelo de ejecución y de toma de decisión, que además suele ser válido y común para todos.

Siguiendo la propuesta de Newell (1986), podemos distinguir tres tipos de constreñimientos:

- Entorno: relacionado con las características del balón, la superficie de juego, las condiciones ambientales, los posibles elementos distractores (público, presencia del entrenador etc.).

- Personales: Araújo \& Volossovitch (2005) nos indican que estos constreñimientos expresarán la tendencia de un jugador a realizar determinadas acciones y están relacionados con las características propias del jugador, tanto a nivel físico o antropométrico (talla, peso, velocidad, fuerza etc.), habilidades técnico-tácticas y las cualidades psicológicas.

- Tarea: relacionadas directamente con las características que presentan las diferentes tareas de entrenamiento. Desde las diferentes reglas de juego, la manipulación de los espacios de juego, las zonas, las metas, el número de jugadores, las limitaciones motrices (número de toques por jugador) y temporales (finalizar a gol antes de 15 segundos), hasta las situaciones competitivas (tanteo en el marcador, puntuación etc.).

Podemos considerar que de los tipos de constreñimientos anteriormente mencionados, dos de ellos (Tarea y Entorno) tienen una relación muy directa con dinámica del juego y su lógica interna. Es por ello, que en el siguiente punto de este trabajo se desarrollará una propuesta práctica de la manipulación de estos elementos para acercar diferentes tipos de tarea a los principios de la dinámica del juego. Pero antes, en la siguiente tabla (Tabla 1) se propondrá una clasificación de diferentes tipos de tareas (Inespecífica $=$ situaciones $\sin$ oposición, oposición pasiva o poca oposición; Genérica = situaciones con un grado medio de oposición, en espacios amplios o reducidos; Modificadas $=$ situaciones con una alto grado de oposición y espacios intermedios-amplios; Reales $=$ situaciones de juego real en espacio de juego máxi-

\begin{tabular}{|c|c|c|c|c|c|}
\hline $\begin{array}{c}\text { Nivel } \\
\text { Complejidad }\end{array}$ & $\begin{array}{l}\text { Tipo de } \\
\text { tarea }\end{array}$ & $\begin{array}{c}\text { Grado } \\
\text { Incertidumbre }\end{array}$ & $\begin{array}{c}\text { Grado de } \\
\text { inestabilidad }\end{array}$ & $\begin{array}{c}\text { Grado } \\
\text { especificidad }\end{array}$ & $\begin{array}{c}\text { Grado } \\
\text { interacción }\end{array}$ \\
\hline I & Inespecífica & * & * & ** & * \\
\hline II & Genérica & *** & ** & *** & *** \\
\hline III & Modificada & $* * * *$ & $* * * * *$ & ****** & $* * * *$ \\
\hline IIII & Real & $* * * * *$ & $* * * * *$ & $* * * * *$ & $* * * * *$ \\
\hline
\end{tabular}

mo o casi máximo, desde 9x9 hasta 11x11) para determinar que grado de incertidumbre, especificidad e inestabilidad muestran cada una de ellas.

Teniendo en cuenta los criterios de la tabla anteriormente expuesta, podemos considerar que las situaciones inespecíficas y genéricas son la menor nivel de complejidad, debido a que son las más alejadas a la realidad del juego y a su relación medio-baja con los principios de la dinámica del juego. En el siguiente punto del trabajo se propondrá una dinámica práctica con el fin de manipular los elementos de la tarea para aumentar en un mayor grado la relación tipo de tarea-principios de la dinámica del juego.

\section{Conclusiones}

Esta nueva concepción sobre el concepto de tarea de entrenamiento, la cual nace desde la visión de la pedagogía no lineal, tendencia que promueve el diseño de tareas como medio para provocar nuevos oportunidades de acción o affordance a través de los conceptos de variabilidad e inestabilidad que ocasionará la auto-organización de las diferentes estructuras del deportista, nos hace considerar y replantear el concepto de tarea de entrenamiento, teniendo un enfoque más dinámico y adaptado a las necesidades de aprendizaje, que el propio tradicional. Es más, podríamos considerar que el diseño de tareas de entrenamiento debería ser entendido como la elaboración de contextos de aprendizajes (Martín-Barrero, 2019; Torrents et al. 2011).

Después de analizar los diferentes elementos y del desarrollo práctico propuesto en este trabajo, sería también interesante, reflexionar acerca de la idea de fomentar constreñimientos/condicionantes que provoquen conductas naturales en el juego, es decir, que no dependan de estímulos no reales del juego (ejemplo el color de un cono o la indicación de una señal inespecífica del entrenador), para ello es importante tener en cuenta los diferentes tipos de constreñimientos y como manipular los mismos para provocar los comportamientos deseados y orientar las tareas a los contenidos de trabajo planteados.

\section{Referencias}

Araújo, D. y Volossovitch, A. (2005). Fundamentos para o treino da tomada de decisão: uma aplicação ao Andebol. En D. Araújo (Ed.), O Contexto da decisão: a acção táctica no desporto (pp. 75-97). Lisboa: Edições Visão e Contextos.

Balagué, N., Pol, R., Torrents, C., Ric, A., \& Hristovski, R. (2019). On the Relatedness and Nestedness of Constraints. Sports Medicine, 5(6), 1-10.

Caldeira, P., Paulo, A., Infante, J. \& Araujo, D. (2019). A influência da pedagogia não-linear e da abordagem baseada nos constrangimentos no treino do remate no voleibol. Retos, 36, 590-596.

Chow, J. Y., Davids, K., Button, C., Shuttleworth, R., Renshaw, I., \& Araújo, D. (2007). The Role of Nonlinear Pedagogy in Physical Education. Review of Educational Research, 77(3), 251-278. https://doi.org/10.3102/003465430305615

Clark, M. E., Mcewan, K., \& Christie, C. J. (2018). The effectiveness of constraints-led training on skill 
development in interceptive sports : A systematic review. International Journal of Sports Science \& Coaching, 14(2), 241-254.

Del Villar, F. \& García, L. (2014). El entrenamiento táctico y decisional en el deporte. Madrid: Síntesis.

Flores-Rodríguez, J. (2019). Pedagogía no lineal en el Balonmano: ideas generales para su aplicación. En Martín-Barrero, A. \& Camacho, P. (2019) Nuevas tendencias en el entrenamiento y la planificación de los deportes colectivos. Sevilla: Wanceulen.

Gamero Portillo, M., García Ceberino, J., Reina Román, M., Feu Molina, S., \& Antúnez Medina, A. (2019). Estudio de las variables pedagógicas de las tareas de baloncesto en función de las fases de juego. Retos, 37(37), 556-562.

Gibson, J. (1979). An Ecological Approach to Visual Perception, Boston: Mifflin.

Lagardera, F. \& Lavega, P. (2003) Introducción a la praxiología motriz. Barcelona: Paidotribo.

Martin-Barrero, A. (2019). El proceso de enseñanza-aprendizaje en el fútbol: aproximación a un enfoque basado en competencias en el fútbol formativo. Sevilla: Wanceulen.

Newell, K. M. (1986). Constraints on the Development of Coordination. In M. G. Wade, \& H. T. A. Whiting (Eds.), Motor Development in Children: Aspects of Coordination and Control (pp. 341-360). The Netherlands: Martinus Nijhoff, Dordrecht. http:// dx.doi.org/10.1007/978-94-009-4460-2_19.

Parlebas, P. (1976). Activités physiques et éducation motrice, 4. EPS, dossiers 1967-1977.

Parlebas, P. (1981). Contribution à un lexique commenté de l'action motrice. Paris: INSEP.

Parlebas, P. (1988). Elementos de Sociología del Deporte. Málaga: Unisport.

Parlebas, P. (2001). Juegos, deportes y sociedad. Léxico de praxiología motriz. Barcelona: Editorial Paidotribo.

Passos, P., Araujo, D., \& Shuttleworth, R. (2008): Manipulating constraints to train decision making in rugby union.
International Journal of Sports Science and Coaching, 3(1), 125-140.

Pic, M. \& Lavega-Burgués, P. (2019). Estimating motor competence through motor games. Revista Internacional de Ciencias de la actividad física y del Deporte, 55, 5-19. doi: 10.5232/ricyde2019.05501

Pic, M. \& Navarro, V. (2017). Triad communication and specificity of motor games. Revista Internacional de Medicina y Ciencias de la actividad ísica y del deporte, 17, 523-539. doi: 10.15366/rimcafd2017.67.009

Pic, M., Lavega-Burgués, P. \& March-Llanes, J. (2019). Motor behaviour through traditional games. Education Studies. 45, 742-755.

Pic, M., Navarro-Adelantado, V. \& Jonsson, G. K. (2018). Detection of ludic patterns in two triadic motor games and differences in decision complexity. Frontiers in Psychology. 8:, 2259. doi: 10.3389/fpsyg.2017.02259

Reina, M., Gamero, M. G., León, K. \& Ibáñez, S. J. (2018). Development and pedagogical structure of training tasks in formative basketball. ESHPA-Education, Sport, Health and Physical Activity, 2(2), 145-161.doi:10481/51752

Reina, M., García Rubio, J., Antúnez, A., \& Ibáñez, S. (2019). Comparación de la carga interna y externa en competición oficial de 3 vs. 3 y 5 vs. 5 en baloncesto femenino Retos, 37(37), 400-405

Torrents, C., Araujo, D., Gordillo, A. \& Vives, M. (2011). El diseño de contextos de enseñanza-aprendizaje para el desarrollo de la motricidad. Tándem. Didáctica de la educación Física, 36, 27-35.

Urgelés, A. (2004) Los dominios de acción motriz como base de los 266 diseños curriculares en educación física: el caso de la Comunidad de Aragón en educación primaria. En Lagartera, F.y Lavega,P.(Ed) La ciencia de la acción motriz. Lleida: Universitat de Lleida (p. 203226).

Warren, W. (2006). The Dynamics of Perception and Action, Psychological Review, 113(2), 358-389.

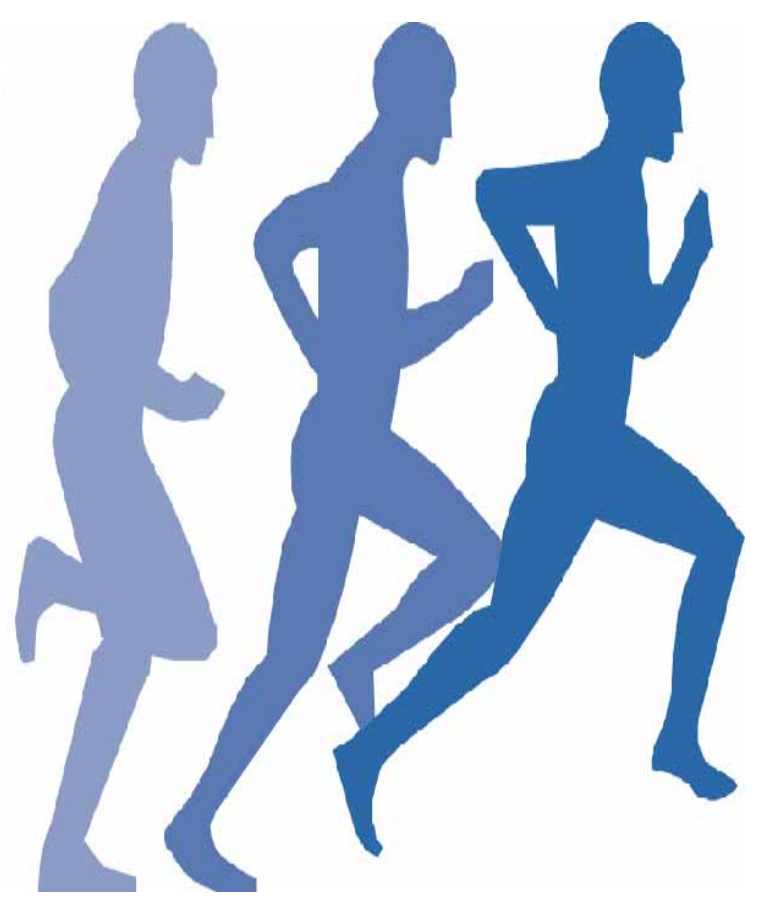

